\title{
AVALIAÇÃO RETROSPECTIVA DOS CASOS DE SÍNDROME DE GRISEL (FIXAÇÃO ROTATÓRIA C1-C2) NO IOT-HCFMUSP
}

\author{
RETROSPECTIVE EVALUATION OF GRISEL'S SYNDROME (C1-C2 ROTATORY FIXATION) AT \\ IOT-HCFMUSP
}

\author{
EVALUACIÓN RETROSPECTIVA DE CASOS DE SÍNDROME DE GRISEL (FIJACIÓN \\ ROTATORIA C1-C2) EN IOT-FMUSP
}

Fabiano Cortese Paula Gomes ${ }^{1}$ Gullherme Pereira Correa Meyer ${ }^{1}$, AleXandre Sadao lutaka², Alexandre Fogaça Cristante ${ }^{3}$, Raphael Martus Marcon ${ }^{3}$, Reginaldo Perilo Oliveira ${ }^{3}$, Tarcísio Eloy Pessoa de Barros Filho ${ }^{4}$

RESUMO

Objetivo: Avaliar o perfil dos casos de fixação rotatória C1-C2, seu tratamento e complicações. Métodos: Estudo retrospectivo de 35 casos com fixação rotatória C1-C2 no período de 1982 a 2008. Foram analisados a idade, sexo, quadro clínico, classificação, tratamento e complicações. Resultados: Dos 35 casos avaliados, 19 eram meninas e 16 meninos, sendo a idade média de 6,9 anos. 0 principal sintoma foi dor (85\%), seguida por deformidade (71\%) e perda de mobilidade cervical (62\%). Foram encontrados de acordo com a classificação Fielding e Hawkins 11 casos tipo I, 14 tipo II e 5 casos tipo III. Nenhum caso foi classificado como tipo IV. Em cinco casos a classificação não pode ser obtida. O tratamento mais utilizado foi o uso do colar cervical (20 casos), seguido pela tração (seis casos) e a artrodese C1-C2 (seis casos). Conclusão: A fixação rotatória C1-C2 é uma doença muito mais frequente na infância, em geral causada por uma contratura do músculo esternocleidomastoideo seguida de uma IVAS. Na maioria dos casos a fixação é reversível somente com tratamento conservador, em especial se o tratamento for precoce. Em casos com evolução tardia, pode ser necessária a tração mentoneira ou craniana e nos casos irredutíveis a artrodese C1-C2.

Descritores: Síndrome; Áxis; Atlas cervical; Criança; Artrodese; Resultado de tratamento.

\begin{abstract}
Objective: To evaluate the profile, treatment and complications of patients with C1-C2 rotatory fixation. Methods: A total of 35 patients with diagnosis of C1-C2 rotatory fixation at IOT-HCFMUSP, between 1982 and 2008, were retrospectively studied. Age, gender, clinical features, classification, type of treatment and complications were evaluated. Results: Of a total of 35 cases, 19 were girls and 16 were boys, with a mean age of 6.9 years. The most common symptom was pain (85\%), followed by deformity (71\%) and loss of cervical mobility (62\%). According to Fielding and Hawkins classification, we found 11 cases type I, 14 cases type II, and 5 cases type III. There were no type IV cases. In 5 cases we couldn't obtain classification. The use of collar was the most used treatment (20 cases). Traction was performed in 6 cases and C1-C2 arthrodesis in 6 cases. Conclusion: C1-C2 rotatory fixation is a disorder more common in children. The cause is a contracture of the sternocleidomastoid muscle, usually after an upper airway tract infection. The fixation is reduced with conservative treatment, mainly with early treatment. When treatment starts late, the use of cervical traction can be necessary and for irreducible cases C1-C2 arthrodesis .
\end{abstract}

Keywords: Syndrome; Axis; Cervical atlas; Child; Arthrodesis; Treatment outcome.

\section{RESUMEN}

Objetivos: Evaluar el perfil de los casos de fijación rotatoria C1-C2, su tratamiento y complicaciones. Métodos: Estudio retrospectivo de 35 casos con fijación rotatoria C1-C2 en el período de 1982 a 2008. Se analizó edad, sexo, cuadro clínico, clasificación, tratamiento y complicaciones. Resultados: De los 35 casos, 19 eran niñas y 16 niños, siendo 6,9 años la edad promedio. El principal síntoma fue dolor (85\%), seguido por deformidad (71\%) y pérdida de movilidad cervical (62\%). Fueron encontrados 11 casos tipo I de Fielding y Hawkins, 14 casos tipo II y 5 casos tipo III. Ningún caso fue clasificado como de tipo IV. En 5 casos, no se pudo obtener la clasificación. El tratamiento más utilizado fue el uso del collar cervical (20 casos), seguido por la tracción (6 casos) y la artrodesis C1-C2 (6 casos). Conclusión: La fijación rotatoria C1-C2 es una enfermedad mucho más frecuente en la infancia, causada generalmente por una contractura del músculo esternocleidomastoideo seguida de una IVAS. En la mayoría de los casos, la fijación es reversible solamente con tratamiento conservador, principalmente si el tratamiento fuere precoz. En casos con evolución tardía, puede ser necesaria la tracción mentoniana o craneana y, en los casos irreductibles, la artrodesis C1-C2.

Descriptores: Síndrome; Axis; Atlas cervical; Tortícolis; Niño; Artrodesis; Resultado del tratamiento

\footnotetext{
1 - Médico Residente (R5) do Grupo de Coluna do Instituto de Ortopedia eTraumatologia do HC/FMUSP - São Paulo, Brasil.

2 - Médico Assistente do Grupo de Coluna do Instituto de Ortopedia eTraumatologia do HC/FMUSP - São Paulo, Brasil.

3 - Doutor, Médico Assistente do Grupo de Instituto de Ortopedia eTraumatologia do HC/FMUSP - São Paulo, Brasil.

4 - Professor Titular do Departamento de Ortopedia da Faculdade de Medicina da USP - São Paulo, Brasil.
}

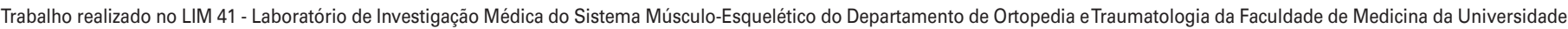
de São Paulo - SP, Brasil.

Correspondência: Rua Dr Luiz Teixeira Mendes, 1833, Zona 5, Maringá - PR, Brasil. CEP. 87015-000 - E-mail: fabianocpgomes@ ortopedista.com.br 


\section{INTRODUÇÃO}

A primeira descrição de fixação rotatória C1/C2 foi feita por Bell ${ }^{1}$ em 1830, em um caso de ulceração da faringe. Em 1930, Grisel descreveu a associação dessa condição com história recente de infecção de vias aéreas superiores (IVAS), tendo atualmente seu nome relacionado a essa associação ${ }^{2,3}$ Essa alteração já teve diversas denominações, como luxação não traumática C1/C2, luxação inflamatória C1/C2, subluxação não traumática C1/C2, fixação rotatória C1/C2. Usaremos nesse artigo o termo fixação rotatória C1/C2.

Muito mais frequente em crianças, a fixação rotatória C1/C2 é, na maioria dos casos, precedida por uma IVAS ou afecção inflamatória do pescoço ou cabeça ${ }^{4-6}$. Quando ocorre em adultos em geral é devido a traumatismos de alta energia, tais como acidentes automobilísticos.

Os principais sintomas da fixação rotatória C1/C2 são dor cervical e perda de mobilidade do pescoço. No exame clínico encontra-se a cabeça inclinada para um lado e com rotação para o lado oposto. À palpação, o músculo esternocleidomastóideo encontra-se relaxado do lado em que a cabeça está inclinada e tensa no lado oposto. Devido a esse fato, a fixação rotatória C1/C2 também é chamada de "torcicolo paradoxal". Outro sinal encontrado no exame físico é o desvio do processo espinhoso de C2 para o mesmo lado da cabeça, chamado de sinal de Sudek.

Os exames de imagem podem auxiliar no diagnóstico. A radiografia cervical transoral pode evidenciar assimetria da articulação C1/C2 ${ }^{7}$ (Figura 1). A rotação entre as vértebras envolvidas também pode ser observada com mais clareza na tomografia computadorizada.

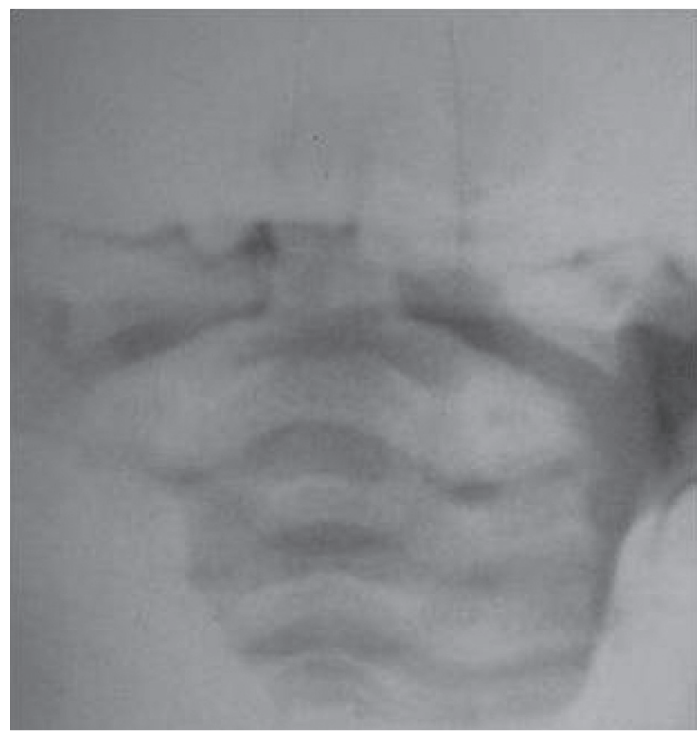

Figura 1. Radiografia cervical transoral com assimetria da articulação C1-C2.

\section{OBJETIVO}

O objetivo deste estudo foi avaliar o perfil dos casos de fixação rotatória C1/C2 e os resultados do tratamento no IOT-HCFMUSP entre os anos de 1982 a 2008.

\section{MATERIAIS E MÉTODOS}

Foram avaliados os casos de fixação rotatória C1/C2 internados no IOT-HCFMUSP entre os anos de 1982 a 2008, sendo um total de 35 casos. Após a análise dos prontuários médicos, foram incluídos no estudo somente os casos com informações completas e que possuíam radiografias. Foram analisados idade, sexo, quadro clíni- co, exames radiológicos, classificação, tratamento e complicações.

A classificação utilizada foi a de Fielding e Hawkins, baseada no sentido e na distância do deslocamento do atlas sobre o áxis ${ }^{8,9}$ (Figura 2).

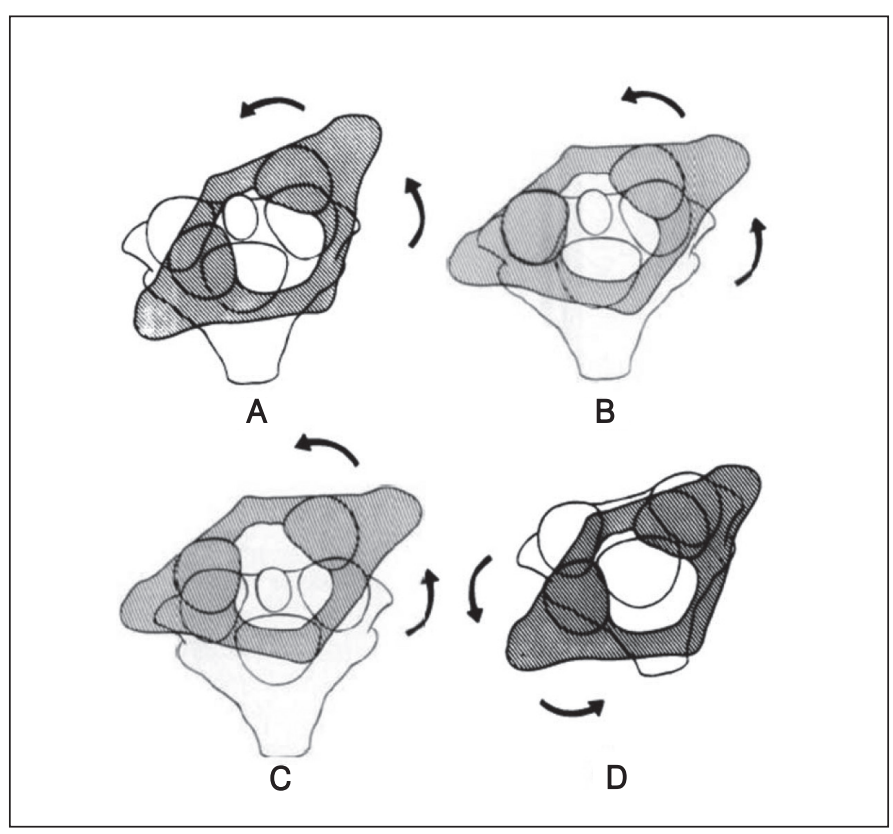

Figura 2. a) Tipo I b) Tipo II c) Tipo III d) Tipo IV.

Tipo I: desvio rotatório do atlas, sem deslocamento anterior. Neste tipo, o desvio ocorre dentro do arco de movimento normal entre C1/C2. Tipo II: desvio rotatório com deslocamento anterior do atlas menor que $5 \mathrm{~mm}$.

Tipo III: desvio rotatório com deslocamento anterior do atlas maior que $5 \mathrm{~mm}$.

Tipo IV: desvio rotatório com deslocamento posterior do atlas sobre o áxis.

\section{RESULTADOS}

Dos 35 casos, 19 eram do sexo feminino e 16 do sexo masculino. A idade média foi de 6,9 anos, variando de três a 14 anos.

A queixa principal foi de dor em 30 casos (85\%). As outras queixas foram deformidade e perda de mobilidade cervical.

O intervalo entre o início dos sintomas e primeira avaliação variou de 8 horas a 140 dias.

História de IVAS prévia recente foi identificada em 28 dos 30 casos (80\%). Também foi encontrada história de trauma cervical recente em cinco casos (14\%), sendo em um deles trauma de alta energia.

Em apenas um caso foi encontrada presença de déficit neurológico. O quadro clínico foi de paresia do deltóide e tríceps ipsilaterais.

Em relação à classificação de Fielding e Hawkins, encontramos 11 casos classificados como tipo I, 14 casos como tipo II e cinco casos como tipo III. Nenhum caso foi classificado como tipo IV. Em cinco casos a classificação não pode se obtida, devido à má qualidade das radiografias.

\section{TRATAMENTO}

O tratamento foi guiado de acordo com a classificação, idade e tempo de evolução (Tabela 1).

Em 21 casos foi utilizado o tratamento com colar cervical ou 
gesso. A tração mentoneira foi utilizada em seis casos, sendo a redução obtida em todos. Em oito casos a redução com métodos conservadores não foi possível, sendo instalados o halo craniano.

O tratamento cirúrgico foi indicado em 6 pacientes, sendo realizada a artrodese C1-C2 (Figura 3). Foi instalado halo craniano pré-operatório, sendo realizada a cirurgia após a redução da deformidade. No pós-operatório os pacientes permaneceram com halogesso por três semanas (Figura 4). Todos os casos apresentaram sinais radiográficos de consolidação da artrodese após 16 semanas de pós-operatório (Figura 5).

Tabela 1. Relação entre a classificação obtida e o tratamento realizado.

\begin{tabular}{c|c|c|c|c|c|c}
\hline Classificação & SC & Tipo I & Tipo II & Tipo III & Tipo IV & Total \\
\hline Gesso & 0 & 0 & 1 & 0 & 0 & 1 \\
\hline Colar & 3 & 8 & 9 & 0 & 0 & 20 \\
\hline $\begin{array}{c}\text { Tração Mentoneira } \\
+ \text { gesso }\end{array}$ & 0 & 1 & 2 & 0 & 0 & 3 \\
\hline $\begin{array}{c}\text { Tração mentoneira } \\
+ \text { colar }\end{array}$ & 1 & 2 & 0 & 0 & 0 & 3 \\
\hline $\begin{array}{c}\text { Halo + Halo gesso } \\
\text { Artrodese C1-C2 }\end{array}$ & 0 & 0 & 2 & 4 & 0 & 6 \\
\hline Total & 5 & 11 & 14 & 5 & 0 & 35 \\
\hline
\end{tabular}

SC: Sem classificação.

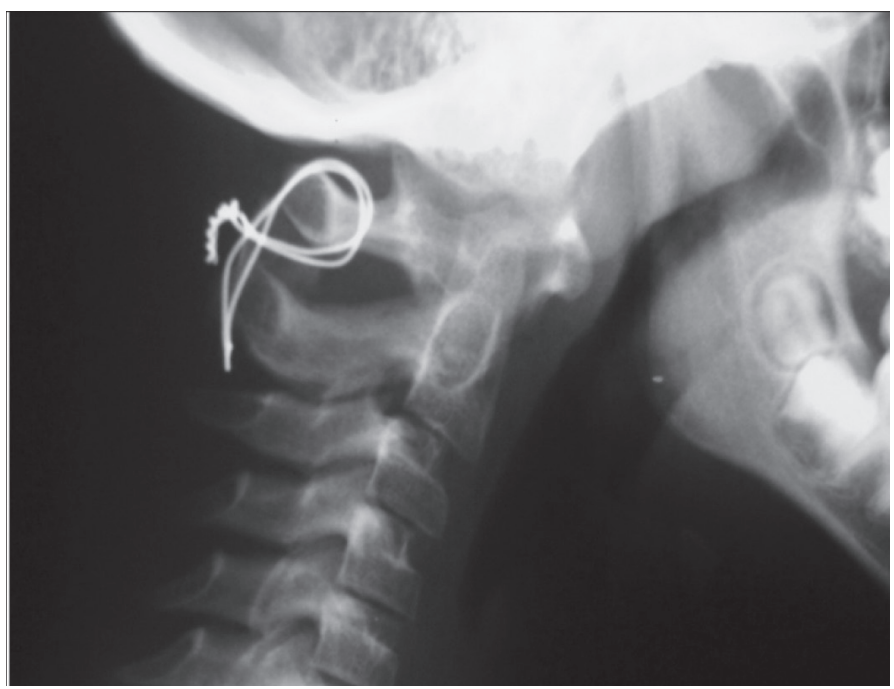

Figura 3. Artrodese C1-C2 com fixação e enxerto estruturado.

\section{COMPLICAÇÕES}

As complicações decorrentes do tratamento foram escassas. Os casos tratados com colar e tração mentoneira não apresentaram complicações.

Em oito pacientes foi utilizado o halo gesso isolado ou no pósoperatório. Dois deles apresentaram infecção no sítio de inserção dos pinos do halo craniano. Os dois casos foram tratados com antibioticoterapia e apresentaram melhora completa do quadro. O paciente que apresentou déficit neurológico obteve resolução espontânea do quadro.

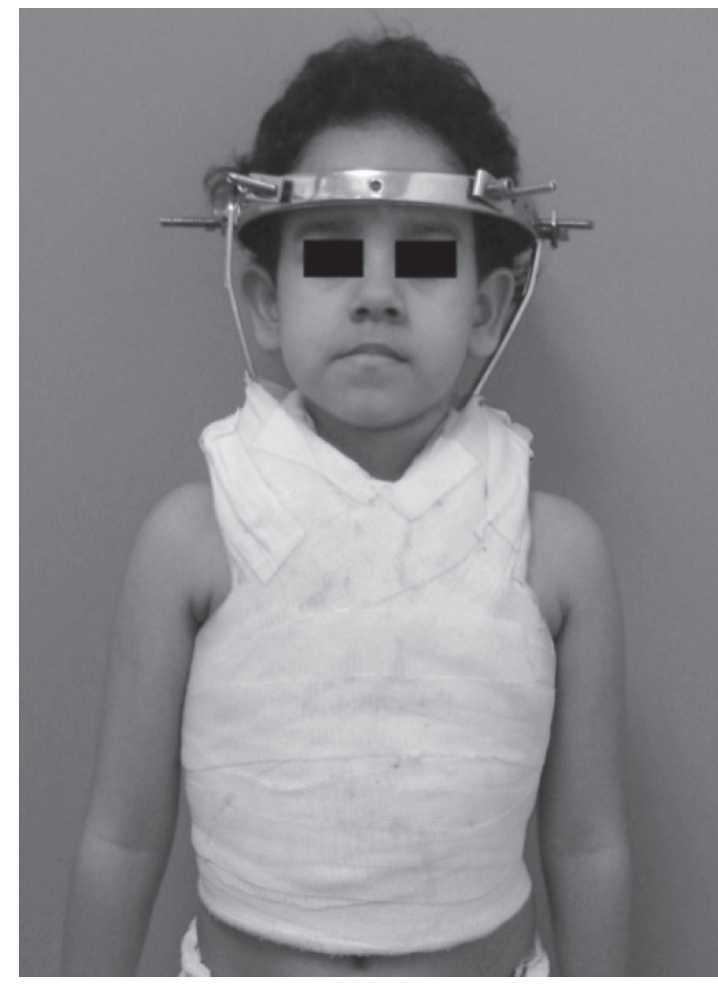

Figura 4. Paciente em uso de halo-gesso.

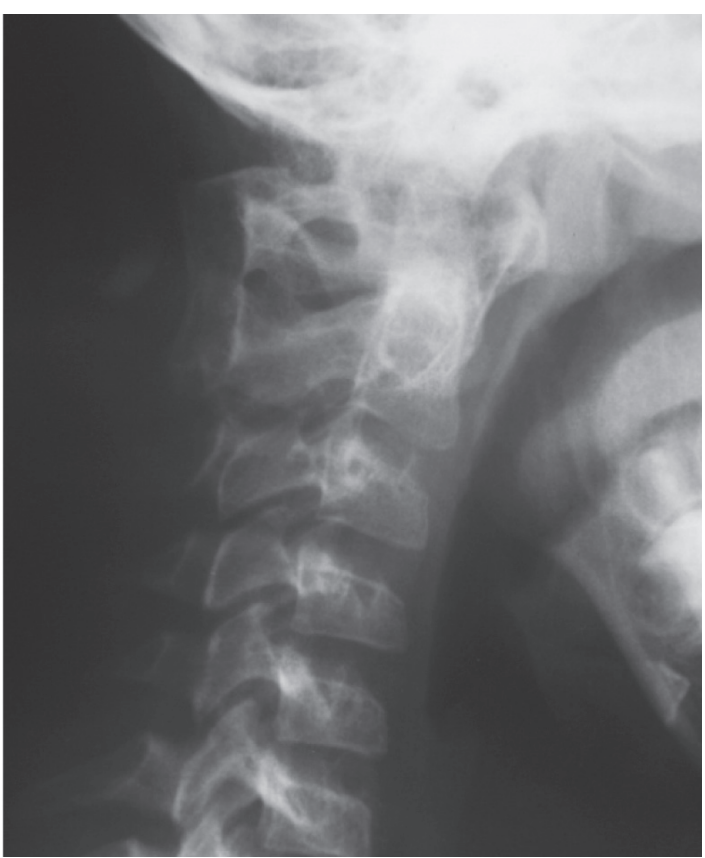

Figura 5. Artrodese C1-C2 consolidada.

\section{DISCUSSÃO}

A fixação rotatória C1-C2 é uma doença de acometimento quase exclusivo da população pediátrica. Watson-Jones ${ }^{10}$ e Wilson e Michele ${ }^{11}$ em séries de casos demonstraram que a maioria dos pacientes acometidos encontra-se na primeira década de vida.

A maioria dos casos apresentou quadro de IVAS prévia ao aparecimento dos sintomas. Entretanto, uma história negativa de IVAS não deve excluir o diagnóstico.

A maioria dos casos foi classificada como tipo I, ou II de Fielding 
e Hawkins. Apenas 5 dos 35 casos foram classificados como tipo III. O tratamento conservador com colar e com tração mentoneira foi realizado em dois casos, com bons resultados.

Em oito (22\%) pacientes foi necessário o tratamento com halo craniano ou artrodese C1-C2. Dos oito casos, cinco foram classificados como tipo III, dois como tipo II e em 1 caso não foi obtida classificação. Os dois casos tipo II apresentaram diagnóstico tardio, um com 93 dias e outro com 119 dias. Fielding e Hawkins ${ }^{8}$ em 1977 apresentou uma série de casos com 17 pacientes com fixação rotatória C1-C2. Em 13 casos (76\%) foi realizado o tratamento cirúrgico com artrodese cervical. A idade média dos casos foi de 20,6 anos e o tempo médio dos sintomas até o diagnóstico foi de 11,6 meses.

\section{CONCLUSÃO}

A fixação C1-C2 é uma doença de evolução benigna na maioria dos casos. O tratamento conservador, com colar cervical ou tração mentoneira é suficiente na maioria dos pacientes. Nos casos em que o desvio da fixação seja mais pronunciado ou quando o diagnóstico é feito tardiamente o uso da tração com halo craniano ou artrodese podem ser necessários.

\section{REFERÊNCIAS}

1. Bell C. The nervous system of the human body. London: Longman; 1830. p. 403

2. Hirth K, Welkoborsky HJ. [Grisel's syndrome following ENT-surgery: report of two cases]. Laryngorhinootologie. 2003;82(11):794-8.

3. Grisel P. Enucleation de l'atlas et torticolis nasopharyngien. Press Med. 1930;38:50-3.

4. Harma A, Firat Y. Grisel Syndrome: nontraumatic atlantoaxial rotatory subluxation. J Craniofac Surg; 2008;19(4):1119-21.

5. Mezue WC, Taha ZM, Bashir EM. Fever and acquired torticollis in hospitalized children. J Laryngol Otol. 2002;116(4):280-4

6. Welinder NR, Hoffmann P, Håkansson S. Pathogenesis of non-traumatic atlanto-axial subluxation (Grisel's syndrome). Eur Arch Otorhinolaryngol. 1997;254(5):251-4.
7. Fernández Cornejo VJ, Martínez-Lage JF, Piqueras C, Gelabert A, Poza M Inflammatory atlanto-axial subluxation (Grisel's syndrome) in children: clinical diagnosis and management.Childs Nerv Syst. 2003;19(5-6): 342-7.

8. Fielding JW, Hawkins RJ. Atlanto-axial rotatory fixation. (Fixed rotatory subluxation of the atlanto-axial joint). J Bone Joint Surg Am. 1977;59(1):37-44.

9. Wetzel FT, La Rocca H. Grisel's syndrome. Clin Orthop Relat Res. 1989;(240):141-52.

10. Watson-Jones R. Spontaneous hyperemic dislocation of the atlas. Proc R Soc Med. 1932;25:586

11. Wilson M, Michele AA, Jacobson EW. Spontaneous dislocation of the atlanto-axial articulation, including a report of a caso with quadriplegia. J Bone Joint Surg 1940:22:698. 